\title{
Subsystems of Electric Vehicle: Overview
}

\author{
Abhinav Agarwal ${ }^{1}$, Abhishek H Chachadi ${ }^{2}$, Akshay Kumar Singh ${ }^{3}$ and Sabit Auti ${ }^{4}$ \\ 1,2,3,4 Electronics and Communication Dept \\ R. V. College of Engineering, \\ Bangalore, India \\ 19abhinavagarwal.ec17@rvce.edu.in , aahishekhc.ec17@rvce.edu.in , \\ 3akshaykumars.ec17@rvce.edu.in and ${ }^{4}$ sabitauti.ec18@rvce.edu.in
}

\begin{abstract}
Not long prior to entering the 20th Century, the most popular sort of transport at this point was the horse. Be that as it may, as individuals' wages expanded and developments progressed, some were starting to explore different ways regarding more up to date types of transport. Presently, gas, steam, and electrical power were all available, with each following strength watching out. Steam development was grounded as of now and was generally seen and trusted by individuals all in all. It had, in light of everything, shown its worth driving assembling plants, mines, gets ready, and conveys - it had all the earmarks of being only a trademark development to collect more unobtrusive kinds of transport using steam engines. There was an issue - steam motors were too slow to warm up and especially during winters it was particularly very tough to start them and once started the person had to continuously supply water for its cooling. They likewise had a restricted reach and should have been continually taken care of with water. Electric vehicles, or EVs for short, work using an electric engine rather than an interior ignition motor, similar to gas fueled vehicles. Much of the time, EVs utilize an enormous footing battery pack to control the engine. This battery pack is charged by being connected to an exceptionally planned charging station or outlet at the clients' home. As EVs run on power, they have no fumes and don't contain parts like the fuel siphon, fuel line, carburetor, and gas tank, which are required in gas-controlled vehicles. But the evidence of the positives has gotten amazingly clear, there are also a couple of hindrances that every individual needs to consider before they choose to make an electric vehicle their next tremendous undertaking. The reasons being: - Recharge Points, The Initial Investment is Steep, Short Driving Range and Short Driving Speed, Not Suitable for Cities, Facing Shortage of Power. To overcome the challenges more and more research and development work has been carried out and most of the above stated challenges have been resolved. Higher battery density for longer range, alternate Li-ion batteries to increase the efficiency and to reduce the initial cost, and powerful chargers for fast charging are going under continuous development. Li-ion batteries undergo performance degradation and cycle ageing, and it needs to be identified as soon as possible, i.e., using a Recommended Architecture to improve the performance of an EV. Apart from these, EV offers an easy and efficient testing and verification model.
\end{abstract}

Keywords: Electric Vehicle, Motor, Battery, Charging, Dynamic Recommendation, Architecture, Performance, Testing

\section{INTRODUCTION}

Electric vehicles are the answer to one of the most alarming problems which is declining petroleum reserves. EVs are also an eco-friendly solution which can help in reducing carbon footprint. Studies are done in the field of automated vehicles to improve its characteristics and make it even better. An Electric vehicle consists of several subsystems for proper functioning. These subsystems are crucial for efficient operation and smooth functioning of the vehicles which are also useful in finding faults and eliminating them to provide a good and safe user experience. Extra features have always attracted the customer which will make it easier to market, several subsystems are designed specifically to target that issue. The most important and 
crucial step before setting up the vehicle for public use is safety and functionality check along with fault detection and analysis. Hence, a safety control system is designed and installed to guarantee user safety. This is done in accordance with all the safety measures and protocols provided by the road and transport governing authorities.

Electric vehicles were first witnessed in the mid-19th century. At that time due to lack of technological advancement they were less effective as compared to the conventional IC engine vehicles. At that time, it had very short range of reach and as well as low top speed, battery life was also less, on top of that it was costly. Outcome of this was worldwide decline of their use. Now from the end of the late 20th century the concern of hydro-carbon emission and declining petroleum reserve was increasing which factored in the starting of research in alternative fuel vehicles. This has made electric vehicles more popular. Companies like Tesla and Google have already made fully electric self-driving cars. There is a lot of research work still required in this field to reach new heights.

This paper investigates the current subsystems present in the Electric vehicles and provides a detailed analysis of each of those subsystems. The study also describes the drawbacks of the present system and finally this paper sheds some light on some new ideas based on the present technology which can be implemented in the future.

\section{Study on features of EVs Background}

The range of EVs is limited so they have to be periodically recharged. To overcome this, some of the solutions are Remote Power Transfer Using Solar Power, Wireless Charging employing Mobile Energy Distributors, Increase the Driving Range by utilizing Wind Energy. It also described the disadvantages associated in implementing solutions to improve the range of electric vehicles.[1]

There are some complexities involved in management of the availability of charging points. The authors suggested how telecommunication companies can help in management of such issues. With the widespread connectivity, telcos can support the planning of the EV charging network. Telcos can also provide analyses of traffic and streams. They can also provide cryptographic communication services to ensure security of vehicles connected to the network.[2]

A risk management framework of Electric Vehicle Charging Stations is suggested. The framework consisted of safety considerations, risk assessment techniques and its management. The work addressed the concern about the hazardous voltages appearing in Electric Vehicle Supply Equipment. It was also shown how Electric Vehicle Charging Stations are threatened by cyber-attacks.[3]

There can be a system that enables electric vehicles to sell electricity back to the power grid. The methodology adopted for this is to recharge EVs when electricity is cheap and trade energy back to the power grid when it is costly. This makes sure that the EVs are charged enough when they need to be used.[4]

Incorporation of an inbuilt charging system of electric vehicles which can charge the vehicles while they are running would be significant improvement. The designed system includes a motor installed on the front shaft which acts as the drive circuit and a generator installed on the rear shaft of the vehicle. The kinetic energy of the rotating shaft is converted into electric energy. This electrical energy charges the battery pack which acts as a source of energy for vehicles.[5]

Smart Brake model for optimized regeneration and also keeping the orientation of the vehicle intact is suggested. The model implements brake blending strategy and employs an algorithm to meet the requirements. Decision of splitting the torque requirement between the regenerative brake and conventional one is carried out by brake blending controller. The algorithm decides to split the brake demand between the electric and hydraulic actuator.[6]

The charging strategy for an EV can decrease the costs and also improve the durability of the intelligent grid network. But there are situations where EVs are influenced by voltage security issues and working expenses. Reducing the power fluctuation level in the grid network and the charging cost for EV users is the main aim. The problem with respect to power 
fluctuation of the network smart grid system that considers EVs battery storage capacities, rate at which EV is charging, charging behavior of EV users is formulated [7]. An AdaptiveBlockchain-Based-EV participation (AdBEV) plot is utilized to reduce the power fluctuation and also reduce charging cost of EVs.

The design format adaptability and execution of the $\mathrm{X}$ by wire (XBW) vehicle, in a XBW electric vehicle, has more prominent defects and must be improved. To improve the above qualities the Control Configured Vehicle (CCV) guideline is executed on a XBW EV. Considering the Electric-Control System together including the mechanical part for the layout, the CCV improves the layout structure flexibility of the XBW vehicle. From the results of the input experiments, it can be seen that yaw control system improves the handling of the vehicle and stability associated with it.[8]

Considering vehicles to network grid applications, vehicles have the ability to control the power. The grid functions are developed using V2 technology. Power systems are more flexible with the integration of EVs. But, the fast growth of EVs will generate several problems in the efficient operation and control of the power system in the upcoming future [9] and can be addressed by integrating EVs with the Microgrid.

The EV-to-EV (V2V) charging enables active cooperation with charging/discharging operations between EVs. A flexible energy management protocol along with different V2V algorithms makes EVs more efficient, flexible. Smart Charging/Discharging behaviors is much needed and hence, this Flexible Management Protocol along with V2V is more efficient. Simulation results verify the improvement of the EV utilities and the Energy Consumption of the EVs using the above-mentionedmethod [10]

EVs with Lithium-ion batteries suffer from many problems like loss of performance and ageing of the battery which is going to be a great problem to the EVs since it's the power source. These problems can be caused due to the effect of the behavior of the EV user. Considering this, a Dynamic Recommendation Architecture is required to provide Recommendations in order to find these flaws as soon as possible and let the EV user know to prolong the life of the battery. Thus, a DRA has been designed and proposed [11]

Testing plays the most crucial role in development and deployment of any product in the industry. Considering the case of connected and automated vehicles (CAVs) or electric vehicles there is a lack of a symmetric framework that can generate a library consisting of all the testing scenarios. The solution to this can be generated by providing a general framework for testing scenarios library generation that is applicable for a variety of operational design domains and performance matrices [12].

With the increase in technological advancement in the field of electric drive vehicles, a new problem of electromagnetic interference (EMI) has risen. There is a need to investigate the electromagnetic compatibility of the electric vehicle according to the European standard which is ECE R10-5. Emission test is done to check the amount of EM waves emitted by the DUT and how it is affecting the surrounding. Immunity test is done to check how the electromagnetic interferences caused by the surroundings like radio waves or health instruments etc., is affecting the DUT [13].

Batteries are the most essential part of an electric vehicle hence, determining the state of health of the battery becomes important with a focus on reliable, safe and cost-effective battery operation. A method is developed to calculate the condition of the battery using a wellestablished figure of merit, 10-s discharge resistance without actually performing the laboratory test [14].

Driver security is the most essential part in the ebb and flow period. With unexpected expansion in urbanization, the quantity of mechanized vehicles on streets have expanded because of the increasing needs of the people. With that numbers of mishaps also increases and to limit the street mishaps, smart helmets are fabricated with inbuilt sensors like IR, gas sensor, load sensor, and 3-pivot accelerometer for recognizing any accidents. With every one of the sensors working consummately, the rider will actually want to turn on the vehicle. The liquor sensor relies upon the distance between sensor and mouth, the degree of liquor is identified in the body. The GPS sensor utilized is in-form ones that are available in the cell phone. Low 
versatile sign, high velocity movement, more established variant GPS modules are the fundamental driver for decreasing GPS precision. With that the 3 - pivot accelerometer had a high precision. It could recognize the accelerometer esteem up to $1.280 \mathrm{~g}$ from the first worth. In the testing time frame, accidents discovery exactness is almost 100\% [15].

Because of fast urbanization, the number of vehicles on the streets have been radically expanding because of which the contamination level likewise increases. What's more, Fossil fuel saves are on the verge of depletion, which is due to great number of IC engine vehicles out and about. Numerous investigations show that mixed electric vehicles are better than ordinary IC motor driven vehicles. The Quick Adoption and Manufacturing of Hybrid and Electric Vehicles (FAME) plan was presented in 2015 by the Government of India to advance crossover and electric vehicles. The HEV do accompanies a few drawbacks, the significant ones are the high introductory speculation cost which may cause issues from the three-wheeler proprietors since most of the individuals are from working class family. A minimal expense retrofit unit for changing traditional IC motor three-wheelers over to half breed electric threewheeler will draw in a great deal of clients as a result of the extensive improvement in vehicle proficiency absent a lot of extra ventures [16].

\section{Research Methodology}

Every insurance policy consists of five parts: declarations, insuring agreements, definitions, exclusions and conditions. These days many policies contain a sixth part: endorsements. Each of these have their own importance which is important tounderstand.

\subsection{Range of EVs}

Electric Vehicles (EVs) are effective, cost less and emit less than Internal Combustion Engines. Many people use EVs to attract attention and not as a real mode of daily transport because of their small range. To overcome this problem, solutions which can improve their range significantly are suggested.

\subsubsection{Remote Power Transfer Using Solar Power}

This solution includes a transmitter and a receiver coil. The coil should safeguard electromagnetic waves from gadgets like portable telephones. These coils are positioned in CAM (Cement Asphalt Mortar) streets. An IR sensor is utilized to distinguish the presence of EV for the charging to occur.

\subsubsection{Wireless Charging employing Mobile Energy Distributors}

This incorporates wireless Charging utilizing mobile energy distributors. This is a device which supplies electricity on the go. Sewer vent covers will be utilized as charging stations and mobile energy distributors are customized to take a course on which the distributors will charge during their drive. Distributors go about as fuel sources to EVs that need charging. The vehicle requiring the charge will move toward the suitable truck.

\subsubsection{Increase the Driving Range by utilizing Wind Energy}

The body of the vehicle can be embedded with a wind turbine. The battery of the EV will be connected to this turbine. When the wind strikes the turbine, it will charge the battery. This can lead to improving the vehicle's range.

\subsection{Braking System of EVs}

The quantities of mishaps occurring on our streets have dramatically expanded in the course of the last not many a long time making the streets an exceptionally perilous spot for both the people on foot just as the riders. In contrast to four-wheelers, the significant issue in regards to two wheelers are the equilibrium factor during the slowing down measure, which if runs wild 
can cause serious issues.

A programmed stopping mechanism has been created which gives exact outcomes and can be basically placed into utilization even in the vehicles for the crippled, where the balance factor is invalidated in view of the extra balance-wheels given. The framework can additionally be carried out in like manner bikes with the assistance of self-adjusting innovation. The Whole System is divided into following units:

\subsubsection{Sensing unit}

Gadgets or modules, whose main role is to distinguish varieties and changes in the climate and pass the data to other electronic gadgets and processors are named as sensors. Significant ones being ultrasonic sensors, Infrared sensors, radars, etc. They find their significance in a few fields including autos, clinical applications, military applications, and so forth.

\subsection{Braking Unit}

Ultrasonic sensors modified by Arduino are set in front of the vehicle to give the separation from any snag inside the predetermined reach. This data is utilized to control the DC engine which pulls the brake switch in the introduced slowing mechanism.

\subsubsection{Alarming Unit}

The fundamental signs to be given is perhaps the most significant thought to keep away from an impact on the street with any impending vehicle, especially from behind. The caution officer comes right into it before the slowing down interaction and capacities as a notice to the driver about a conceivable impact that could occur except if a momentary move is made and furthermore to show to the driver behind conceivable slowing down to stay away from additional crashes from behind.

\subsection{Battery Technology of EVs}

The most expensive part in an EV is their Lithium-ion batteries, a huge segment of the expense of such a vehicle notwithstanding the way that their expense is dropping due to improvements. They have higher life expectancy, more efficient and have high energy density. While having a few benefits, batteries degrade and as time goes on and use, which impedes their performance. In like manner, Li-ion batteries should provide safety and reliability as well as reduce degradation of cells. Due to usage behavior EV users get affected by battery degradation. Thus, client actions which degrade the battery in an unconventional way must be recognized. To identify the battery degradation and its ageing we need a Recommendation Architecture helping the EV user to increase the life of a Battery.

A Dynamic Recommendation Architecture (DRA) is required to create dynamic suggestions to the EV user. It is a design dependent on logical decisions. The information stream and fundamental logic is utilized to execute this DRA. We get Recommendations from previous driving data and this information is stored utilizing an On-Board Diagnostics to Bluetooth connector. Further information sources are utilized, which are GPS just as a charging station and the board framework. These give data about driven courses and insights about performed charging measures at various charging stations.

EV receives the GPS data on an android tablet, which is utilized as a transmitting node between time series and on-board diagnostics buffer in the recommendation engine. The dynamic recommendation architecture takes raw time data from the sources and stores them in a buffer. Preprocessing module receives the buffered data and the data is abstracted. Charging as well as usage behavior is obtained.

\subsection{Testing of EVs:}

A testing subsystem is dedicated to check the vehicle before deployment.The subsystems generate a testing scenario library which is applicable for a variety of operational design 
domains and performance matrix. A four-step process is used to attain the required general framework.

First the most important step is Scenario Description, which includes testing scenario parameterization along with decision variables definitions. This is done by listing all the factors which are influencing the testing scenario.

Secondly, Metric Design is done. Performance metrics are defined for determining the aspects responsible for vehicle evaluation. This paper focuses on functionality, mobility, comfort and most importantly rider's safety. Performance index estimation is done to quantify all the performance metrics. For e.g., for safety performance Accident rate is calculated and for functionality performance failure rate is evaluated.

Third step deals with Library Generation for a given performance metric. The idea is to formulate a significance function which assigns an importance value to a given testing scenario. The testing scenario will only be included in the library if its importance value exceeds a certain threshold. Here the significance of the testing scenario is defined by the criticality measure. Criticality measure consists of exposure frequency (estimated from Naturalistic Driving DATA, NDD) and Maneuver challenge.

Lastly the CAV evaluation is done by it with the generated testing scenario library. This further includes three steps: Sampling testing scenario from the library then, performing the test and estimating the performance indices from the result.

\section{Discussion}

As the range was discussed in the previous section, a significant increase in it can be achieved if the inbuilt infrastructure and hardware is improved. A motor consists of different types of losses. If the quality of winding is improved, one can reduce the losses and improve efficiency. Also, by improving the storage technology i.e., by using Hydrogen Fuel Cells and Ultracapacitors a good increase in the efficiency can be achieved. Ultra- capacitors have faster response time and Hydrogen Fuel cells have high energy densities. All these benefits combined, efficiency and range of the electric vehicles can be drastically improved.

The Dynamic Recommendation Architecture provides the data which is processed and it is received using an OBD using Bluetooth Adapter, instead a cloud database can be created and can be stored additionally. Later on, GPS data is sent to android Tablet, but if it is stored in a cloud database, we can access data from mobile devices or Personal computers and process them accordingly. Thus, making DRA much more efficient and extensible from the previous DRA proposed [12].

Testing plays a very important role in manufacturing and deployment of any industrial products. A subsystem dedicated entirely for this will definitely make things more organized and efficient. To automate the whole testing process one efficient way is to connect all the measurement and testing devices over a network. Different devices are used to imitate the different parts of a vehicle for testing, like programmable power supply is used to imitate throttle control action of an actual electric vehicle. Simulation of a real world on road testing becomes easier when all the devices are connected over a single network. An application program can easily control the testing procedure with the help of testing scenario library generation [13]. Automation in testing will save time and increase accuracy as there is less occurrence of human error.

\section{Conclusion}

It is evident that EV are the future of transport and travel. From manufacturing till deployment of the vehicle there are several subsystems involved to make it even better. Battery plays the most important role replacing the conventional source of fuel i.e., petroleum. Recharging and battery cycle comes into picture, Dynamic Recommendation Architecture is required with cloud database to identify the Battery degradation and cycle ageing in order to determine the battery flaws and correct them as soon as possible. Additionally fault detection is crucial in EVs hence a subsystem is dedicated solely for the same. This dedicated subsystem provides automation in testing which increases the accuracy of testing and decreases time to market. On road testing is costly hence simulation of real-world scenarios will somewhat reduce the overall cost. In future, there is a scope to eliminate on road testing and perform simulation on 
real world testing scenarios.

\section{REFERENCES}

\subsection{Journal Articles}

[1] P.Kadav and Z. D. Asher, "Improving the Range of Electric Vehicles," 2019 Electric Vehicles International Conference (EV), (2019), pp. 1-5, doi: 10.1109/EV.2019.8892929.

[2] G. Zaffiro and G. Marone, "Smart Mobility: new roles for Telcos in the emergence of electric and autonomous vehicles," 2019 AEIT International Conference of Electrical and Electronic Technologies for Automotive (AEIT AUTOMOTIVE), (2019), pp. 1-5, doi: 10.23919/EETA.2019.8804575.

[3] B. Wang, P. Dehghanian, S. Wang and M. Mitolo, "Electrical Safety Considerations in Large-Scale Electric Vehicle Charging Stations," in IEEE Transactions on Industry Applications, vol. 55, no. 6, pp. 6603-6612, Nov.Dec. (2019), doi: 10.1109/TIA.2019.2936474.

[4] N. K. Breum, M. N. Joergensen, C. A. Knudsen, L. B. Kristensen and B. Yang, "A Charging Scheduling System for Electric Vehicles using Vehicle-to-Grid," 2019 20th IEEE International Conference on Mobile Data Management (MDM), (2019) pp. 351- 352, doi: 10.1109/MDM.2019.00-36.

[5] U. Gupta and D. K. Yadav, "Inbuilt Charging System of Electric Vehicles through Generator Installed on the Rear Shaft of the Vehicle," 2019 Global Conference for Advancement in Technology (GCAT),(2019), pp. 1-4, doi: 10.1109/GCAT47503.2019.8978338.

[6] L. Pugi, T. Favilli, L. Berzi, E. Locorotondo and M. Pierini, "Application of Regenerative Braking on Electric Vehicles," 2019 IEEE International Conference on Environment and Electrical Engineering and 2019 IEEE Industrial and Commercial Power Systems Europe (EEEIC / I\&CPS Europe),(2019), pp. 1-6, doi: 10.1109/EEEIC.2019.8783318.

[7]. C. Liu, K. K. Chai, X. Zhang, E. T. Lau and Y. Chen, "Adaptive Blockchain-Based Electric Vehicle Participation Scheme in Smart Grid Platform," in IEEE Access, vol. 6, pp. 25657-25665, (2018), doi: 10.1109/ACCESS.2018.2835309.

[8] J. Ni, J. Hu and C. Xiang, "Control-Configured-Vehicle Design and Implementation on an X-by-Wire Electric Vehicle," in IEEE Transactions on Vehicular Technology, vol. 67, no. 5, pp. 3755-3766, May (2018), doi: 10.1109/TVT.2018.2805886.

[9] S. Iqbal et al., "Aggregated Electric Vehicle-to-Grid for Primary Frequency Control in a Microgrid- A Review," 2018 IEEE 2nd International Electrical and Energy Conference (CIEEC), (2018), pp. 563-568, doi: 10.1109/CIEEC.2018.8745952.

[10] R. Zhang, X. Cheng and L. Yang, "Flexible Energy Management Protocol for Cooperative EV-to-EV Charging," in IEEE Transactions on Intelligent Transportation Systems, vol. 20, no. 1, pp. 172-184, Jan. (2019), doi: 10.1109/TITS.2018.2807184.

[11] M. Eider and A. Berl, "Dynamic Generation of Recommendations for EV Battery Health," 2018 International Conference of Electrical and Electronic Technologies for Automotive, (2018), pp. 1-6, doi: 10.23919/EETA.2018.8493182.

[12] S. Feng, Y. Feng, C. Yu, Y. Zhang and H. X. Liu, "Testing Scenario Library Generation for Connected and Automated Vehicles, Part I: Methodology," in IEEE Transactions on Intelligent Transportation Systems, vol. 22, no. 3, pp. 1573-1582, Mar. (2021), doi: 10.1109/TITS.2020.2972211.

[13] M. D. Kozan and E. Usta, "EMC Test Requirements for Electric Vehicles," 2019 Fifth International Electromagnetic Compatibility Conference (EMC Turkiye), (2019), pp. 1-3, doi: 10.1109/EMCTurkiye45372.2019.8976016.

[14] G. Giordano, V. Klass, M. Behm, G. Lindbergh and J. Sjöberg, "Model-Based Lithium-Ion Battery Resistance Estimation From Electric Vehicle Operating Data," in IEEE Transactions on Vehicular Technology, vol. 67, no. 5, pp. 3720-3728, May (2018), doi: 10.1109/TVT.2018.2796723.

[15] M. A. Rahman, S. M. Ahsanuzzaman, I. Rahman, T. Ahmed and A. Ahsan, "IoT Based Smart Helmet and Accident Identification System," 2020 IEEE Region 10 Symposium (TENSYMP), (2020), pp. 14-17, doi: 10.1109/TENSYMP50017.2020.9230823.

[16] S. Sudhakaran, S. Indulal and L. P. P. S, "Fuzzy Logic Controller Design for Retrofitted Three-wheeled Hybrid Electric Vehicle," 2020 First IEEE International Conference on Measurement, Instrumentation, Control and Automation (ICMICA), (2020), pp. 1-5, doi: 10.1109/ICMICA48462.2020.9242654. 